\title{
OntoSlug: a dynamic visual front-end program for ontologies
}

\author{
Martin Telefont ${ }^{1, *}$ and Yunkai Liu ${ }^{2}$ \\ ${ }^{1}$ Department of Biology, University of South Dakota, Vermillion, SD, USA; ${ }^{2}$ Department of Computer Science, University of South \\ Dakota, Vermillion, SD, USA; Martin Telefont ${ }^{*}$ - E-mail: Martin.Telefont@usd.edu; ${ }^{*}$ Corresponding author \\ received May 28, 2008; revised July 17, 2008; accepted July 18, 2008; published July 30, 2008
}

\begin{abstract}
:
The display of ontological information has become a crucial factor over the last decade in systems biology. The possibility to compare different ontological systems in a single application has however not been answered with an appropriate application. OntoSlug is an easy to use application that tries to fill this need. OntoSlug has been developed for use in classroom settings and scientific laboratory environment.
\end{abstract}

Keywords: ontology; dynamic visualization; graphic user interface; biology

\section{Background:}

The recent advances in scientific processing in genetics and proteomics have created an increased demand for the implementation of gene-protein and protein-protein interaction databases for an easy comparison between model organisms [1]. Ontologies have been used to standardize the names and definitions of genes and proteins and represent the relationships among them. Recent efforts in improving the visualization of ontologies [2-4] have demonstrated the value of dynamic visualization. Interaction networks are crucial to our understanding of these systems since they allow for easy abstraction and visualization of complex processes. A good portion of publically available ontologies use customized XML files to store information. Most ontology centered applications are able display and or manipulate data of this complexity, however, lack the possibility of visual information display that would make an easy comparison between different systems or data-sets possible. Most of the available applications are restricted to specific markup language formats instead of a friendly and integrated interface. These factors among others have contributed to a split of biological fields into ones that rely heavily on data visualization [5-7] and others, more traditional research, that uses these tools infrequently.

\section{Features and functionalities:}

OntoSlug can be used to display and fusion of various preexisting ontological systems (Figure 1). Interaction with the data takes mainly place over the GUI which employs four main concepts to display relationships: nodes, links, tags and layers. While node and links are not new concepts, the special function of dynamic joining of nodes between different systems, makes the overlaps between systems more compact without loss of information. The flexible categorization of interactions between elements allows for a quick and flexible adaptation of data that may be present in multiple data collections. This preserving the uniqueness of the data set that could be lost in imported in a static input format.

OntoSlug allows for the rapid import of information from a variety of data sources (using existing data filters) or custom tailored filters that allow for a side by side or overlay/joining of data nodes. Manipulation of the 2D elements requires little or no prior knowledge of the system or the operation of the input/output application. The Graphical User Interface is designed to facilitate the manipulation of nodes, the underlying information they represent and an intuitive display of relationships among them (Figure 2).

The joining function allows for a visual fusion of elements of different data sets. It can be employed from a patch file or manually at the node. Links that link nodes adapt to the new joined node. A split of joined nodes into individual nodes is also possible via patch or manual interaction. The tag option allows for a grouping of nodes into clusters that share a common characteristic, but are not associated via links. The display of a tag group happens via fading to a more transparent shade of coloring, a pulsing highlight or an altered coloring scheme, all of which allow for an easy discrimination of nodes from other nodes not included in the group. Layers, allow the display of entire ontologies, cross sections of ontologies, tag groups, node types and link types. The graphic output of the information is dynamic which allows for an easy arrangement of information and fast adaptability without the need to adjust node arrangement after altering interactions. Furthermore the integration of advanced sorting, comparison, display and modeling tools in OntoSlug, as well as the 


\section{www.bioinformation.net}

\section{Software}

possibility of integrating custom macros, will be an integral part of the next release.

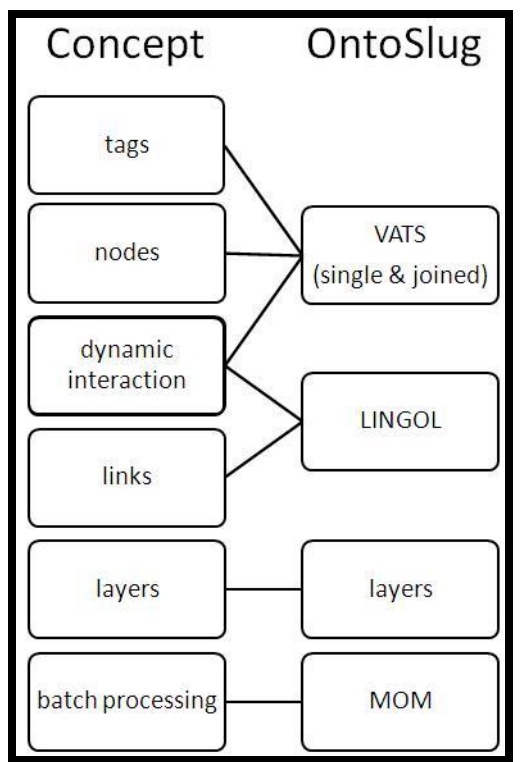

Figure 1: Representation of concepts usage in Ontoslug.

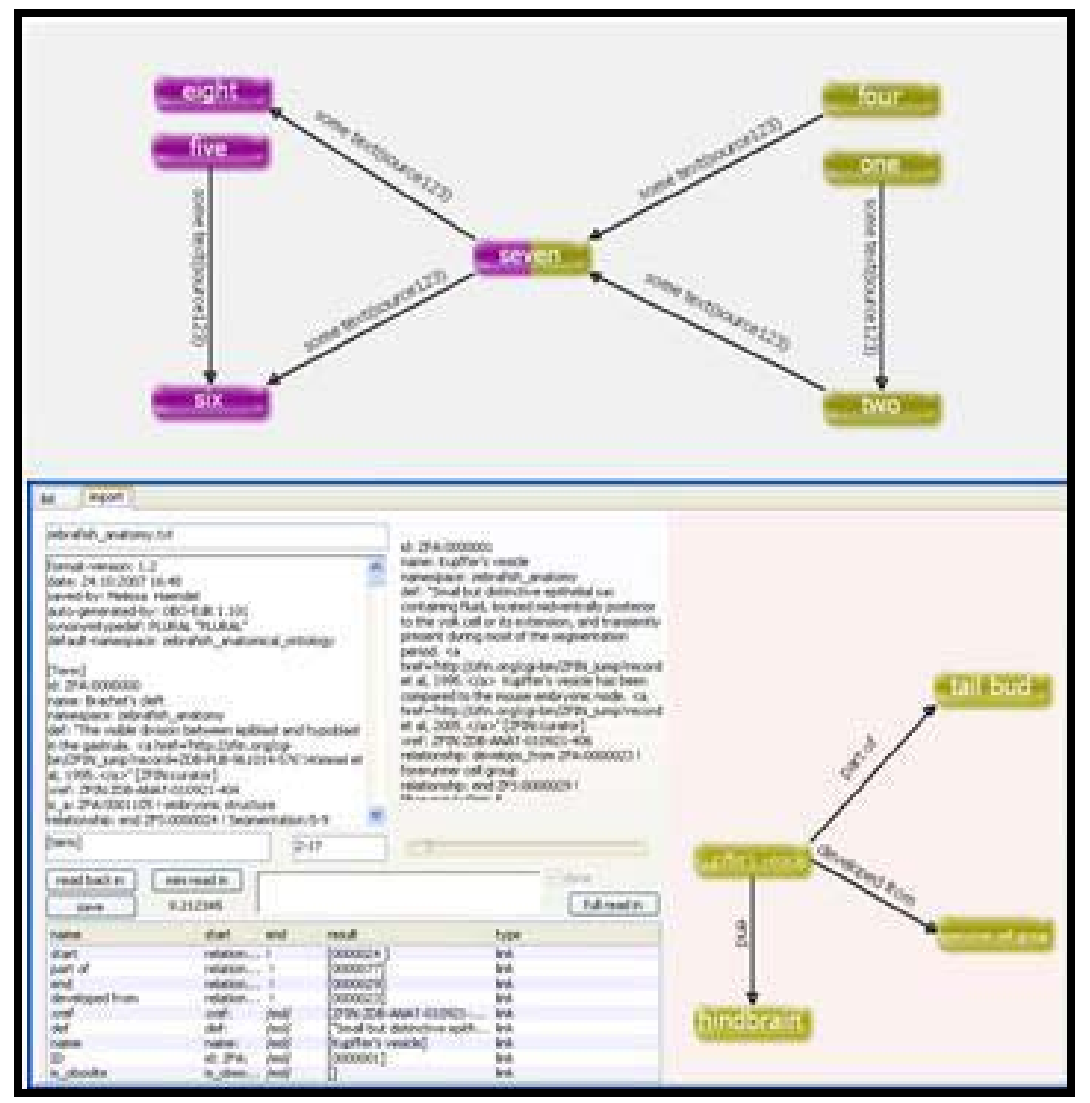

Figure 2: The Graphical User Interface in Ontoslug is shown.

ISSN 0973-2063

Bioinformation 2(10): 438-440 (2008)

439 


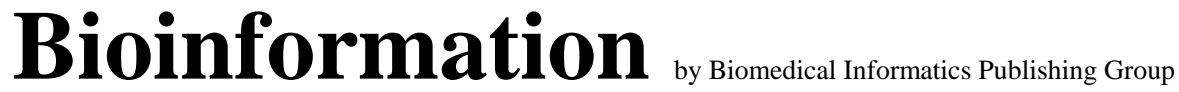

open access

\section{www.bioinformation.net}

Software

\section{Conclusion:}

In conclusion, OntoSlug permits research data/ideas to be easily shared among collaborators and a quick and dynamic adaptation to preexisting interaction paradigms. The ease of data display makes OntoSlug especially useful for utilization in a teaching environment where a modular approach to complex topics is used, while still allowing the fusion of independent topics into an interconnected web of interactions. OntoSlug allows for a cross-platform release (Mac and Linux) given demand. The software is available at www.martintelefont.net/OntoSlug.html.

\section{Acknowledgement:}

This project was supported by NIH Grant Number 2 P20 RR016479 from the INBRE Program of the National Center for Research Resources and a subproject from the NIH National Center for Research Resources grant P20 RR015567 which is designed as a Center of Biomedical Research Excellence (COBRE). We would like to thank Dr. Kenneth
Renner for reviewing the manuscript. MT specially thanks Na Feng for the conceptual support.

\section{References:}

[01] R. Sharan, Proc Natl Acad Sci USA., 102: 1974 (2005) [PMID: 15687504]

[02] L. Salwinski, Bioinformatics, 23: 2193 (2007) [PMID: 17553858]

[03] C. Rohl, Mol Biotechnol., 34: 69 (2006) [PMID: 16943573]

[04] I. Vastrik, Genome Biol., 8: R39 (2007) [PMID: 17367534]

[05] K. Baerenfaller, Science, 320: 938 (2008) [PMID: 18436743]

[06] D. Park, Nucleic Acids Res., 36: W491 (2008) [PMID: 18448467]

[07] J. Klein, Nucleic Acids Res., 36: W460 (2008) [PMID: 18440972]

Edited by P. Kangueane

Citation: Telefont and Liu, Bioinformation 2(10): 438-440 (2008)

License statement: This is an open-access article, which permits unrestricted use, distribution, and reproduction in any medium, for non-commercial purposes, provided the original author and source are credited. 